\title{
Phlyctenular eye disease in association with Hymenolepis nana in Egypt
}

\author{
M. KHALAF Al-HUSSAINI, R. KHAlifA, ${ }^{1}$ ABDEL TAWAB A. Al-ANSARY, \\ GAMAL H. HUSSAIN, AND ABEL KADER M. MOUSTAFA
}

From the ${ }^{1}$ Departments of Ophthalmology and Parasitology, Faculty of Medicine, Assiut University, Assiut, Egypt

SUMMARY It had been previously noticed that infection with parasites was common in children suffering from phlyctenulosis. In the present study the stools of 471 patients suffering from phlyctenular disease were examined and it was found that $62.6 \%$ of them had Hymenolepis nana ova in their stools as compared with $10.8 \%$ of the controls. All patients had Hymenolepis nana immune sera. Many of these patients had abdominal symptoms. Hymenolepis nana is a cestode parasite discovered by Bilharz in Cairo in 1851. Infections with it have the same age incidence and geographical distribution as phlyctenular eye disease. It has a tissue stage responsible for a state of hypersensitivity which is thought to be responsible for the phlyctenules.

Phlyctenular keratoconjunctivitis is a disease occurring mainly in children, with a slight preponderance of males. In Egypt it is very common, constituting about $20 \%$ of cases of acute conjunctivitis (AlHussaini and Saoudi, 1968). It is known to be an allergic response of the corneal and conjunctival epithelium to an endogenous toxin. The most accepted theory was that this toxin is a tuberculoprotein. In $1968 \mathrm{Al}-$ Hussaini and Saoudi found that tuberculoprotein does not seem to be a factor in phlyctenular eye disease in Egypt. Bakly (1929) attributed a high incidence of phlyctenular eye disease in Port-Said, Egypt, to ascariasis. Jeffery (1955) found that Ascaris lumbricoides, Ancylostoma duodenale, Enterobius vermicularis, and Entamoeba histolytica are responsible for a number of eye diseases, phlyctenulosis being the most common.

Al-Hussaini et al. (1977) found that phlyctenular eye disease in Egypt is often associated with intestinal infection with Hymenolepis nana (the dwarf tape worm). A careful examination of the stools of 155 cases of phlyctenular eye disease showed that $57.4 \%$ of them had $H$. nana ova in their stools. In cases of multiple or recurrent phlyctens the eggs were found in $73.4 \%$ of cases.

That preliminary study, pointing to $H$. nana having an important role in the aetiology of

Address for reprints: Professor M. Khalaf Al-Hussaini, Department of Ophthalmology, Faculty of Medicine, Assiut University, Assiut, Egypt phlyctenular eye disease, suggested the need for more evidence. The stools of a larger number of cases were therefore examined, the serum was tested for antibodies, and a specific anthelminthic drug was given to some patients.

\section{Material and methods}

This study includes 471 cases of phlyctenular eye disease, while 157 cases in the same age groups were used as controls. The age and sex, the social condition, and the presence of any abdominal symptoms were noted. The stools of both groups were examined by both the direct and the more sensitive flotation methods (Faust et al., 1975).

In addition to the examination of stools of another 64 cases of phlycten their sera were tested for any circulating antibodies. The sera of 16 controls were also tested. About $2 \mathrm{ml}$ of blood were taken into a closed sterile test-tube. This was left in the slanting position until serum was separated. With sterile Pasteur pipettes serum was pipetted into sterile small vials, which were preserved at $-4^{\circ} \mathrm{C}$ until being used.

Living $H$. nana worms were obtained from the small intestines of laboratory white rats or mice. The test was done after leaving the sera to warm at room temperature. Serum of every case was added separately to few segments of the living strobila of the worm. Immune sera affect adult worms by 
Table 1 Incidence of different parasites in phlycten cases and controls

\begin{tabular}{|c|c|c|c|c|c|c|c|c|}
\hline & \multicolumn{4}{|c|}{ Total number of cases of phlyctenular disease (471) } & \multicolumn{4}{|c|}{ Controls (157) } \\
\hline & Male & Female & Total & $\%$ & Male & Female & Total & $\%$ \\
\hline Hymenolepis nana & 179 & 116 & 295 & 62.63 & 10 & 7 & 17 & $10 \cdot 82$ \\
\hline Ancylostoma duodenale & 14 & 4 & 18 & $3 \cdot 8$ & 3 & 0 & 3 & 1.91 \\
\hline Enterobius vermicularis & 8 & 17 & 25 & $5 \cdot 8$ & 2 & 1 & 3 & 1.91 \\
\hline Strongyloides stercoralis & 0 & 0 & 0 & 0.0 & 0 & 0 & 0 & 0.0 \\
\hline Ascaris lumbricoides & 0 & 1 & 1 & $0 \cdot 21$ & 0 & 0 & 0 & 0.0 \\
\hline Trichostrongylus colubriformis & 0 & 1 & 1 & $0 \cdot 21$ & 0 & 0 & 0 & 0.0 \\
\hline Giardia lamblia & 2 & 1 & 3 & 0.63 & 1 & 0 & 1 & 0.63 \\
\hline
\end{tabular}



Fig. 1 Incidence of different parasites in phlycten cases

completely enveloping them in a layer of precipitate and rupturing the cuticle (Heyneman and Welsh, 1959).

Two phlyctenular cases who were found to be strongly infected with $H$. nana were admitted to hospital. They were given Niclosamide (Yomesan), which is the anthelmintic drug specific for $H$. nana, without the use of steroids or any other topical application to the eyes. In the period of study only these 2 patients accepted admission to hospital; treatment as outpatients is unreliable.

\section{Results}

Table 1 and Fig. 1 show the result of stool examination of 471 patients suffering from phlyctenular eye disease and 157 controls. Out of 471 cases 295 had $H$. nana ova in their stools, or $62.63 \%$, as compared with 17 out of 157 controls, or $10.82 \%$. There was no significant difference between the incidence of other parasites in phlyctenular eye cases and controls (Figs. 1 and 2). Of 295 cases of phlycten 260 had

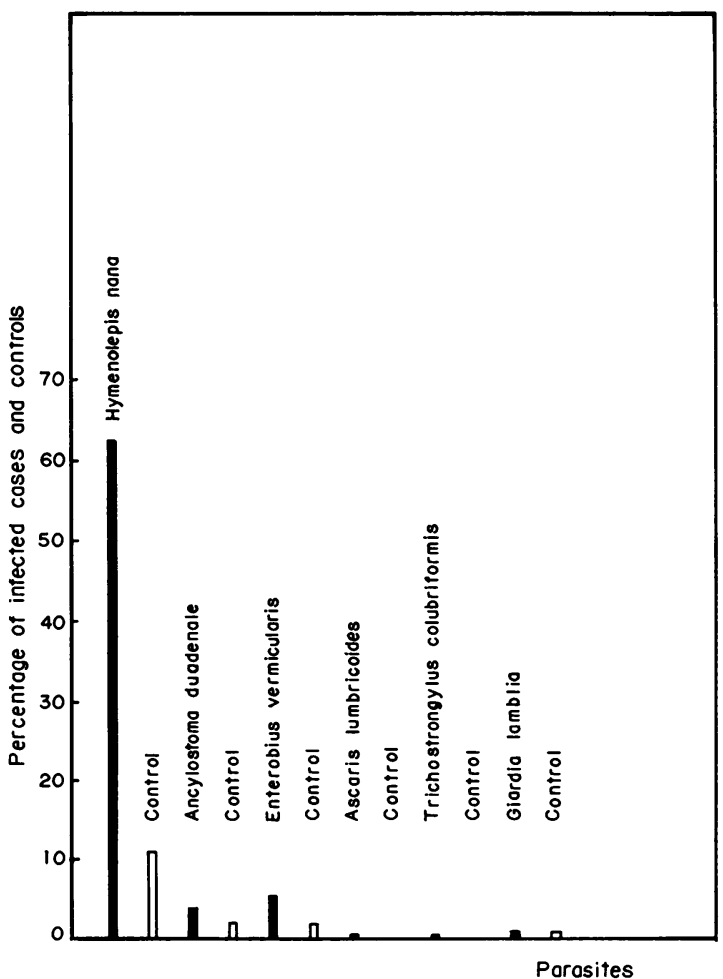

Fig. 2 Percentage of infection with different parasites in phlycten cases and controls 
Table 2 Incidence of Hymenolepis nana ova in cases with multiple phlyctens

\begin{tabular}{lllll}
\hline & $+v e$ H. nana & $-v e$ H. nana & Percentage \\
\hline Multiple phlycten (84) & 63 & 21 & 75 \\
\hline
\end{tabular}

Table 3 Age incidence of cases of phlycten

\begin{tabular}{lll}
\hline Age $($ years $)$ & Number of cases & Percentage \\
\hline $1-10$ & 266 & 56.48 \\
$11-20$ & 149 & 31.63 \\
Over 20 & 56 & 11.89 \\
Total & 471 & \\
\hline
\end{tabular}

Table 4 Age incidence of cases of Hymenolepis nana

\begin{tabular}{lcc}
\hline Age $($ years $)$ & Number of cases & Percentage \\
\hline $1-10$ & 189 & $60 \cdot 58$ \\
$11-20$ & 96 & $30 \cdot 77$ \\
Over 20 & 27 & $8 \cdot 65$ \\
Total & 312 & \\
\hline
\end{tabular}

$H$. nana ova only in their stools; the remaining 35 had mixed infection mostly with Ancylostoma duodenale and Enterobius vermicularis.

Multiple infection with phlyctenules was found in 84 cases; 63 of them had H. nana ova in their stools, or $75 \%$ (Table 2 ).

The age incidence of patients with phlyctenular eye disease is shown in Table 3 , and that of patients with $H$. nana eggs in their stools in Table 4 and Fig. 3.

Both stool and serum examinations were done on 64 phlyctenular cases. The ova of $H$. nana were found in the stools of only 38 of these patients, while a serum test for circulating antibodies showed that all the patients had $H$. nana immune sera. Within a few minutes an enveloping layer was formed round the living segments, which sooner or later ruptured. Although the test was not done quantitatively, it was noticed that the reaction was more powerful and occurred more rapidly in cases of mutliple phlyctenules. As a control the sera and stools of 16 phlycten-free cases were tested in a similar manner. Although one control case was harbouring ova of $H$. nana in the stools, the serum of all the 16 controls failed to give any reaction round the living segments of worms (Table 5).

The 2 inpatients who were given niclosamide (Yomesan) for treatment of $H$. nana were cured of the symptoms of phlyctenular keratoconjunctivitis within a few days without the use of any other systemic or topical remedies.

Many patients gave a history of vague abdominal symptoms in the form of diarrhoea and colic.

\section{Discussion}

Hymenolepis nana was discovered by Bilharz in 1851 in the small intestine of a native boy at necropsy in Cairo, Egypt. It affects $10 \%$ of children in Egypt and Sudan (Belding, 1952). 62.63\% of patients with phlyctenular eye disease had $H$. nana ova in their stools. This incidence rises to $75 \%$ among patients with multiple phlyctens. The eggs of other parasites were significantly fewer than those of $H$. nana. The ova of $H$. nana are not easily detected microscopically. Hence the most careful search should be done

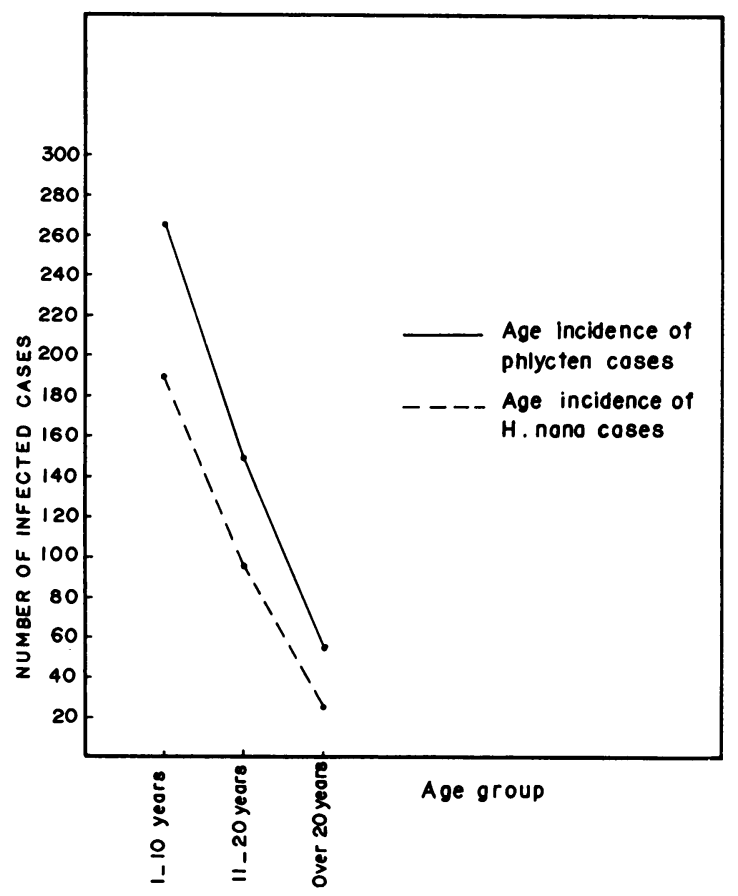

Fig. 3 Age incidence of phlycten cases and $\mathrm{H}$. nana cases

Table 5 Results of serum and stools examination of phlycten and control cases

\begin{tabular}{lllllr}
\hline & $\begin{array}{l}\text { Number of } \\
\text { cases }\end{array}$ & $\begin{array}{l}\text { Positive } \\
\text { stools }\end{array}$ & $\%$ & $\begin{array}{l}\text { Positive } \\
\text { serum }\end{array}$ & $\%$ \\
\hline Phlycten & 64 & 38 & $53 \cdot 1$ & 64 & 100 \\
Control & 16 & 1 & 6.2 & 0 & 0 \\
\hline
\end{tabular}


by a trained observer by both the direct and flotation methods.

The life cycle of $H$. nana in the human body was described in our preliminary report (Al-Hussaini et al., 1977). Most important in this respect is the presence of a tissue stage of the parasite. The larval stages (cysticercoids) penetrate into the villi of the small intestine, where they complete their maturation for about 7 days before they return into the lumen of the intestine. As the parasite is not restricted to the gut, the degree to which it comes into contact with tissue fluids and serum of the host is increased. The development of helminths from the embryonic and larval stages to the sexually mature forms must include biochemical and physiological changes, all of which make the antigenic mosaic increasingly complex (Sinclair, 1970). Several workers have shown that different stages of the worms can elicit different types of antibodies in the host which are to some degree stage-specific, but it is also known that, at least for some helminths, 1 stage is as efficient as another in stimulating immune bodies.

Sinclair (1970) stated that little is known about enzymes which might help the parasite in its invasion of the host tissue, and consequently even less is known about the antibodies which these might stimulate. However, Bogitsh (1967) demonstrated esterases and an alkaline phosphatase in $H$. nana cysticercoids. As more is learnt about the type and location of these enzymes in the parasite and the sequence in which they are found in the life cycle, their effect on the immunological status of the host will become better understood.

Although little success has been obtained in vaccinating animals with dead helminthic material, Coleman et al. (1968) found that $H$. nana homogenate when injected into mice confers a strong immunity. This is further evidence that $H$. nana is an antigenically strong parasite.

The absence of ova in the stools of some patients with phlyctenular eye disease can be explained by intermittent passage of eggs and by the fact that an allergic response to helminths may persist for a long time after disappearance of the parasite from the body. Moreover, helminths may enhance allergic reactions to unrelated antigens (Ershov et al., 1974). The determining factor for the development of phlyctens is not the presence of ova in the stools but the state of hypersensitivity induced by the cysticercoid stage of the parasites. On the same grounds we can explain why some controls have had eggs of $H$. nana in their stools although they did not complain of eye phlyctenules.

The eggs of Ascaris were found in only 1 case during the present study. Bearing in mind that the eggs of Ascaris are easily seen in the stools because of their great number and their conspicuous appearance and knowing that Ascaris is a parasite that has no tissue stage, we consider that Ascaris plays no role in phlyctenular eye disease.

Ancylostoma was found in $3.8 \%$ of cases and in $1.91 \%$ of the controls. The general incidence is low, and the difference between the cases and controls is insignificant. Moreover, Ancylostoma was often associated with $H$. nana in the same patient.

Enterobius vermicularis is best diagnosed by a perianal swab and not by stool examination. In view of the fact that it is a very widely spread parasite among Egyptian children as compared with the incidence of phlyctens among them, and that it is an intestinal parasite that has no tissue stage, it is very unlikely to be responsible for the development of phlyctens.

Amoebiasis is excluded as a factor because it is not associated with eosinophilia or any allergic manifestation (Chandler, 1970).

Abdominal complaints were common among children with phlyctenular eye disease, though accurate statistical data of symptoms definitely related to $H$. nana were impossible because of the prevalence of bowel disturbances of various origins among Egyptian children.

\section{Conclusions}

All cases of phlyctenular eye disease had $H$. nana immune sera. Most of them have $H$. nana ova in their stools. Phlyctenules and infection with $H$. nana have the same age incidence and geographical distribution. Abdominal symptoms are common in patients with phlyctens. Two cases of phlycten eye disease responded dramatically to specific anthelmintic treatment. Other parasites are excluded. It is therefore obvious that the state of hypersensitivity caused by the cysticercoid stage of $H$. nana is responsible for the development of phlyctenules in Egypt.

\section{References}

Al-Hussaini, M. K., and Saoudi, M. S. (1968). The aetiology of phlyctenular eye disease. Proceedings of the Fourth Afro-Asian Congress of Ophthalmology, pp. 425-431.

Al-Hussaini, M. K., Khalifa, R., Ansary, A. T. A., Hussain, G. H., and Mustafa, A. K. (1977). A parasitic factor in phlyctenular eye disease (preliminary note). Bulletin of the Ophthalmological Society of Egypt, 70, 331-338.

Bakly, M. A. (1929). Phlyctenular conjunctivitis in Port-Said. Bulletin of the Ophthalmological Society of Egypt, 25, 57-59.

Belding, D. L. (1952). Textbook of Clinical Parasitology, pp. 559-565. Appleton-Century-Crofts: New York.

Bogitsh, B. I. (1967). Histochemical localization of some enzymes in cysticercoides of two species of Hymenolepis. Experimental Parasitology, 21, 373-379.

Chandler, A. C. (1970). Introduction to Parasitology, pp. 6777. Wiley: New York. 
Coleman, R. M., Carty, J. M., and Graziadei, P. (1968). Immunogenicity and phylogenetic of tapeworm antigens produced by Hymenolepis nana and Hymenolepis diminuta. Immunology, 15, 297-304.

Ershov, V. S., Beresko, V. K., Kashinsky, A. D., Naumytcheva, M. I., and Nisenbaum, I. A. (1974). In Parasitic Zoonosis by Soulby. Academic Press: New York. Faust, E. C., Beaver, P. C., and Jung, R. C. (1975). Animal Agents and Vectors of Human Disease, pp. 422-428. Lea \& Febiger: Philadelphia.
Heyneman, D., and Welsh, J. F. (1959). Action of homologous antiserum in vitro against life-cycle stages of Hymenolepis nana, the dwarf mouse tapeworm. Experimental Parasitology, 8, 119-128.

Jeffery, M. Pauline (1955). Ocular diseases caused by nematodes. American Journal of Ophthalmology, 40, 41-52.

Sinclair, I. J. (1970). The relationship between circulating antibodies and immunity to helminthic infections. In Advances in Parasitology, 8, 97-138. Edited by B. Dawes. Academic Press: New York. 